\title{
Mass splittings within heavy baryon isospin multiplets in chiral perturbation theory
}

\author{
Feng-Kun Guo ${ }^{\mathrm{a} *}$ Christoph Hanhart ${ }^{\mathrm{a} \dagger}$, and Ulf-G. Meißner ${ }^{\mathrm{a}, \mathrm{b}} \neq$ \\ a Institut für Kernphysik and Jülich Center for Hadron Physics, \\ Forschungszentrum Jülich, D-52425 Jülich, Germany \\ ${ }^{\mathrm{b}}$ Helmholtz-Institut für Strahlen- und Kernphysik (Theorie) and Bethe Center for Theoretical Physics, \\ Universität Bonn, D-53115 Bonn, Germany
}

\begin{abstract}
We calculate the mass splittings within the heavy baryon isospin multiplets $\Sigma_{c(b)}$ and $\Xi_{c(b)}^{\prime}$ in chiral perturbation theory to leading one-loop order. The pattern of the mass splittings in the $\Sigma_{c}$ iso-triplet, which is different from that of any other known isospin multiplet, can be explained. We predict $m_{\Xi_{c}^{\prime+}}-m_{\Xi_{c}^{\prime 0}}=-0.2 \pm 0.6 \mathrm{MeV}, m_{\Xi_{b}^{\prime \prime}}-m_{\Xi_{b}^{\prime-}}=-4.0 \pm 1.9 \mathrm{MeV}$ and the mass of the $\Sigma_{b}^{0}$ to be $5810.3 \pm 1.9 \mathrm{MeV}$.
\end{abstract}

Keywords: Chiral Lagrangians; heavy baryons; isospin violation

PACS: 12.39.Fe; 14.20.Lq; 14.20.Mr

\section{Introduction}

Mass splittings within isospin multiplets of hadrons appear due to both the mass difference between the $u$ and $d$ quarks and electromagnetic (em) effects. Since the $d$ quark is heavier than the $u$, usually the hadron with more $d$ quarks is heavier within one isospin multiplet. For instance, the neutron $(u d d)$ is heavier than the proton $(u u d)$, and the $K^{0}(d \bar{s})$ is heavier than the $K^{+}(u \bar{s})$. There is only one exception to this pattern, the $\Sigma_{c}$ iso-triplet, consisting of the $\Sigma_{c}^{++}(c u u)$, the $\Sigma_{c}^{+}(c u d)$ and the $\Sigma_{c}^{0}(c d d)$. The mass splittings within the $\Sigma_{c}$ iso-triplet are measured [1]

$$
\begin{aligned}
& \Delta_{1 c} \equiv m_{\Sigma_{c}^{+}}-m_{\Sigma_{c}^{0}}=-0.9 \pm 0.4 \mathrm{MeV} \\
& \Delta_{2 c} \equiv m_{\Sigma_{c}^{++}}-m_{\Sigma_{c}^{0}}=0.27 \pm 0.11 \mathrm{MeV} .
\end{aligned}
$$

Remarkably, the state with two $u$ quarks has the largest and the one with a $u$ and a $d$ quark has the smallest mass.

Only recently some of the bottom cousins of the $\Sigma_{c}$, the $\Sigma_{b}^{ \pm}$, were observed by the CDF Collaboration [2]. Their masses are for the buu state $m_{\Sigma_{b}^{+}}=5807.8 \pm 2.7 \mathrm{MeV}$ and for the bdd state

\footnotetext{
*Email address: f.k.guo@fz-juelich.de

${ }^{\dagger}$ Email address: c.hanhart@fz-juelich.de

${ }^{\ddagger}$ Email address: meissner@itkp.uni-bonn.de
} 
$m_{\Sigma_{b}^{-}}=5815.2 \pm 2.0 \mathrm{MeV}$, respectively - their neutral partner $\Sigma_{b}^{0}$ has not been observed yet. Thus, here the natural ordering of the states seems to be restored. On the other hand, heavy quark symmetry relates baryons containing a $b$ quark to those with a $c$ quark, which makes this different pattern even more puzzling. In this work we investigate the origin of these patterns, together with those in the $\Xi_{c(b)}^{\prime}$ doublets, using chiral perturbation theory (CHPT) and heavy quark symmetry. In this way we can include both sources of isospin violation in a way consistent with QCD [3]. Our work is a straightforward extension of analogous studies for the nucleon [4, 5] (for pioneering studies, see [6, 7]).

The isospin splittings for heavy baryons were already studied in various quark models in Refs. 8 , 9, 10, 11, 12, 13, 14, 15, 16. The advantages of our investigation are that $(i)$, since we use an effective field theory, the theoretical uncertainty can be estimated, (ii) for the first time meson loop corrections are considered - they turn out to be numerically significant for the charm baryons, and (iii) this study investigates all isospin splittings at the same time. We compare our results to those of the quark models below.

Our paper is organized as follows. In Section 2, we construct the SU(3) chiral effective Lagrangians responsible for the mass corrections of the sextet heavy baryons at order $\mathcal{O}\left(p^{2}\right)$. Here, $p$ denotes the expansion parameter of the underlying effective field theory. The strong Lagrangian is proportional to the quark mass and the em Lagrangian is constructed including virtual photons. As we will show, the operator structure of the effective field theory for the heavy-light baryons is richer than the one of the light quark sector since heavy and light quarks must be treated differently. Calculations up to $\mathcal{O}\left(p^{3}\right)$ are performed in Section 3, A brief summary is given in the last section. Some technicalities are relegated to the appendices.

\section{The chiral effective Lagrangians}

In CHPT the quark mass difference enters explicitly through the quark mass matrix. The inclusion of the virtual photons has been first considered systematically for the three-flavor case in Ref. [17. Chiral Lagrangians with virtual photons have been constructed for the study of isospin symmetry breaking phenomena in mesons and baryons with light up and down quarks (see, e.g., [4, 5, 18, 19, 20, 21, 22, 23, 24, 25, 26, 27, 28, 29] for an incomplete list). Recently, this technique was used to study the interaction between Goldstone bosons and heavy-light mesons and the isospin breaking decay width of the $D_{s 0}^{*}(2317)$ [30].

In this paper, we use the technique of $\mathrm{SU}(2)$ chiral perturbation theory to study the mass splittings within the heavy baryon isospin multiplets. The $u, d$ quark masses and the electric charge $e$ are counted as small quantities. They are booked as $m_{u}, m_{d} \sim \mathcal{O}\left(p^{2}\right)$, and $e \sim \mathcal{O}(p)$ as usual, where $p$ denotes a small momentum with respect to the typical hadronic scale of about $1 \mathrm{GeV}$. Both types of isospin-violating effects $\sim\left(m_{u}-m_{d}\right)$ and $\sim e$ are taken into account here in a systematic manner to the order $\mathcal{O}\left(p^{3}\right)$. These effects can be accounted for in three-flavor CHPT to study the mass splittings within the SU(3) multiplets systematically. However, similar to the case of CHPT for light baryons, the SU(3) breaking contributions from the kaon-baryon and eta-baryon loops are large, see e.g. 31, 32, 33, which makes the convergence of the chiral expansion problematic. We will therefore treat each heavy baryon isospin multiplet separately to $\mathcal{O}\left(p^{3}\right)$ in two-flavor CHPT, and relate the low-energy constants (LECs) to $\mathcal{O}(p)$ and $\mathcal{O}\left(p^{2}\right)$ through $\mathrm{SU}(3)$ relations (see also the discussion in Section 3). On the other hand, the splittings within the multiplets are well behaved. This procedure is equivalent to starting from the $\mathrm{SU}(3)$ Lagrangian and to calculate only the leading order $\mathrm{SU}(2)$ loops, i.e., the pion-baryon loops.

In this section, we will construct the $\mathrm{SU}(3)$ chiral effective Lagrangians pertinent to the mass corrections of the heavy baryons to order $\mathcal{O}\left(p^{3}\right)$ (for similar works considering the strange quark 
as heavy, see Refs. [34, 35, 36, 37]).

In order to construct the chiral effective Lagrangians, the following building blocks are necessary (we employ the standard nonlinear realization of chiral symmetry)

$$
\begin{aligned}
U & =\exp \left(\frac{\sqrt{2} i \phi}{F_{\pi}}\right), \quad u^{2}=U, \\
u_{\mu} & =i u^{\dagger} \nabla_{\mu} U u^{\dagger}, \\
\nabla_{\mu} U & =\partial_{\mu} U-i Q A_{\mu} U+i U Q A_{\mu}, \\
\chi_{+} & =u^{\dagger} \chi u^{\dagger}+u \chi u, \\
Q_{ \pm} & =\frac{1}{2}\left(u^{\dagger} Q u \pm u Q u^{\dagger}\right),
\end{aligned}
$$

where $F_{\pi}$ is the pion decay constant $11, \phi$ collects the Goldstone boson fields

$$
\phi=\left(\begin{array}{ccc}
\frac{1}{\sqrt{2}} \pi^{0}+\frac{1}{\sqrt{6}} \eta & \pi^{+} & K^{+} \\
\pi^{-} & -\frac{1}{\sqrt{2}} \pi^{0}+\frac{1}{\sqrt{6}} \eta & K^{0} \\
K^{-} & \bar{K}^{0} & -\frac{2}{\sqrt{6}} \eta
\end{array}\right),
$$

and $A_{\mu}$ is the photon (em) field. The diagonal quark mass matrix and the charge matrix are

$$
\begin{aligned}
\chi & =2 B_{0} \cdot \operatorname{diag}\left\{m_{u}, m_{d}, m_{s}\right\}, \\
Q & =e \cdot \operatorname{diag}\{2 / 3,-1 / 3,-1 / 3\},
\end{aligned}
$$

in terms of $B_{0}=|\langle 0|\bar{q} q| 0\rangle| / F_{\pi}^{2}$ and the elementary electric charge $e(e>0)$. Under $\mathrm{SU}(3)_{L} \times \mathrm{SU}(3)_{R}$, $u_{\mu}, \chi_{+}$and $Q_{ \pm}$transform as

$$
\mathcal{O} \rightarrow h \mathcal{O} h^{\dagger}
$$

where the compensator field $h$ is an element of the conserved vector subgroup $S U(3)_{V}$.

The $\Sigma_{c}$ iso-triplet and the $\Xi_{c}^{\prime}$ iso-doublet belong to the symmetric sextet and the $\Lambda_{c}^{+}$and the $\Xi_{c}$ doublet belong to the anti-symmetric triplet in the flavor $\mathrm{SU}(3)$ classification. We use the following matrix representation in accordance with the notation of Refs. [38, 39]

$$
B_{6 c}=\frac{1}{\sqrt{2}}\left(\begin{array}{ccc}
\sqrt{2} \Sigma_{c}^{++} & \Sigma_{c}^{+} & \Xi_{c}^{\prime+} \\
\Sigma_{c}^{+} & \sqrt{2} \Sigma_{c}^{0} & \Xi_{c}^{\prime 0} \\
\Xi_{c}^{\prime+} & \Xi_{c}^{\prime 0} & \sqrt{2} \Omega_{c}^{0}
\end{array}\right), \quad B_{\overline{3} c}=\left(\begin{array}{ccc}
0 & \Lambda_{c}^{+} & \Xi_{c}^{+} \\
-\Lambda_{c}^{+} & 0 & \Xi_{c}^{0} \\
-\Xi_{c}^{+} & -\Xi_{c}^{0} & 0
\end{array}\right) .
$$

Under $\mathrm{SU}(3)_{L} \times \mathrm{SU}(3)_{R}$, the transformation laws of the charmed baryon fields are 38.

$$
B_{6 c} \rightarrow h B_{6 c} h^{T}, \quad B_{\overline{3} c} \rightarrow h B_{\overline{3} c} h^{T},
$$

where $h^{T}$ is the transpose of $h$. The matrices for the bottom baryons can be obtained replacing $c$ by $b$ and decreasing the electric charge of every state by one unit.

Let $B_{\overline{3} Q}, B_{6 Q}(Q=c, b)$ denote the heavy baryon fields, and $\stackrel{\circ}{m}_{\overline{3} Q}, \stackrel{\circ}{m}_{6 Q}$ their masses in the chiral limit, respectively. Analogous to the effective chiral Lagrangian for the pion-nucleon system, the lowest order Lagrangian involving the sextet and anti-triplet heavy baryon fields can be written as 38,40 ,

$$
\begin{aligned}
\mathcal{L}^{(1)}= & \frac{1}{2}\left\langle\bar{B}_{\overline{3} Q}\left(i \gamma_{\mu} \tilde{D}^{\mu}-{\stackrel{\circ}{m_{3}}}_{\overline{3}_{Q}}\right) B_{\overline{3} Q}\right\rangle+\left\langle\bar{B}_{6 Q}\left(i \gamma_{\mu} \tilde{D}^{\mu}-{\stackrel{\circ}{m_{6 Q}}}\right) B_{6 Q}\right\rangle \\
& +\frac{1}{2} g_{1}\left\langle\bar{B}_{6 Q} \gamma_{\mu} \gamma_{5} \tilde{u}^{\mu} B_{6 Q}\right\rangle+\frac{1}{2} g_{2}\left\langle\bar{B}_{6 Q} \gamma_{\mu} \gamma_{5} \tilde{u}^{\mu} B_{\overline{3} Q}\right\rangle+\text { h.c. }+\frac{1}{2} g_{3}\left\langle\bar{B}_{\overline{3} Q} \gamma_{\mu} \gamma_{5} \tilde{u}^{\mu} B_{\overline{3} Q}\right\rangle .
\end{aligned}
$$

\footnotetext{
${ }^{1}$ Strictly speaking, this should be the pion decay constant in the chiral limit. To the accuracy we are working, however, we do not need to differentiate this from its physical value.
} 
with

$$
\tilde{D}_{\mu}=D_{\mu}-i Q_{B+} A_{\mu}, \quad \tilde{u}_{\mu}=u_{\mu}-2 Q_{B-} A_{\mu} .
$$

The chiral covariant derivative on the baryon fields $D_{\mu}$ is given by

$$
\begin{aligned}
D_{\mu} & =\partial_{\mu}+\Gamma_{\mu}, \\
\Gamma_{\mu} & =\frac{1}{2}\left(u^{\dagger} \partial_{\mu} u+u \partial_{\mu} u^{\dagger}\right) .
\end{aligned}
$$

The charge matrix of the heavy baryons $Q_{B}$, which gives the correct minimal coupling of the heavy baryons to photons, is constructed as

$$
Q_{B}=2 Q+q_{h} \mathbb{I}= \begin{cases}e \cdot \operatorname{diag}\{2,0,0\}, & \text { for the charm baryons, } \\ e \cdot \operatorname{diag}\{1,-1,-1\}, & \text { for the bottom baryons }\end{cases}
$$

where $q_{h}$ is the charge of the heavy quark, and $\mathbb{I}$ is a $3 \times 3$ unit matrix. $Q_{B \pm}$ is defined as

$$
Q_{B \pm}=\frac{1}{2}\left(u^{\dagger} Q_{B} u \pm u Q_{B} u^{\dagger}\right)
$$

At $\mathcal{O}\left(p^{2}\right)$ the strong Lagrangian pertinent to the corrections of the masses is given by the terms containing one power of the quark mass matrix

$$
\mathcal{L}_{\text {str. }}^{(2)}=-\left\langle\bar{B}_{Q}\left(\alpha_{1} \chi_{+}+\alpha_{2}\left\langle\chi_{+}\right\rangle\right) B_{Q}\right\rangle .
$$

The em Lagrangian at $\mathcal{O}\left(p^{2}\right)$, parameterizing hard virtual photons, is more complicated than the one of the pion-nucleon system [4, 5. The terms, which contribute to the mass corrections of the sextet heavy baryons and are quadratic in the light quark charge matrix read

$$
\begin{aligned}
\mathcal{L}_{Q Q}^{(2)}= & -F_{\pi}^{2}\left\langle\bar{B}_{6 Q}\left[\beta_{0}\left(Q_{+}^{2}-Q_{-}^{2}\right)+\beta_{1} Q_{+}\left\langle Q_{+}\right\rangle+\beta_{2}\left\langle Q_{+}^{2}-Q_{-}^{2}\right\rangle+\beta_{3}\left\langle Q_{+}^{2}+Q_{-}^{2}\right\rangle\right] B_{6 Q}\right\rangle \\
& -F_{\pi}^{2} \beta_{4}\left\langle Q_{+}^{T} \bar{B}_{6 Q} Q_{+} B_{6 Q}\right\rangle .
\end{aligned}
$$

The em Lagrangian given above only deals with the hard photons exchanged between the light quarks in the heavy baryons. In addition we need to add terms that parameterize the em interactions between the heavy and a light quark. Since the heavy quark can be viewed as static, its charge $q_{h}$ acts as a static background field that transforms as a scalar under $\mathrm{SU}(3)_{L} \times \mathrm{SU}(3)_{R}$. It is easy to see that in this way we get one additional independent structure that contributes to the isospin splittings, namely

$$
\mathcal{L}_{\mathrm{em}}^{(2)}=\mathcal{L}_{Q Q}^{(2)}-F_{\pi}^{2} \beta_{1 h}\left\langle\bar{B}_{6 Q} Q_{+}\left\langle q_{h} \mathbb{I}\right\rangle B_{6 Q}\right\rangle .
$$

As we will show below, it is this term which makes the mass splitting pattern within the $\Sigma_{c}$ isotriplet different from that within the $\Sigma_{b}$ iso-triplet. The analogous mechanism is also present in the mentioned quark model calculations.

\section{Mass splittings within the heavy baryon isospin multiplets}

As mentioned above, our strategy is to relate the LECs for different heavy baryon isospin multiplets using $\mathrm{SU}(3)$ relations, but, since we are only after isospin splittings, calculate only the pion-baryon loop corrections. If we start from the $\mathrm{SU}(2)$ Lagrangians for each of the isospin multiplets, the chiral limit masses of the $\Sigma_{Q}$ and $\Xi_{Q}^{\prime}$ are different, and the difference contributes to the $\mathrm{SU}(3)$ 
mass splittings of $\mathcal{O}\left(p^{3}\right)$. It is important to stress that the differences of the LECs between their $\mathrm{SU}(2)$ and $\mathrm{SU}(3)$ values starts contributing at order $\mathcal{O}\left(p^{4}\right)$ and thus is beyond the accuracy of our calculation 2 Therefore, what we do is equivalent to use the $\mathrm{SU}(3)$ Lagrangians given in Section 2 to do $\mathrm{SU}(2)$ calculations. Indeed, the number of independent operators matches for the two cases, since the contribution of the $\beta_{1}$ term to the baryon masses vanishes for $\mathrm{SU}(3)$ because the threeflavor quark charge matrix is traceless, and the $\beta_{0}$ term for $\mathrm{SU}(3)$ can be translated into the $\beta_{1}$ term for $\mathrm{SU}(2)$ by using the Cayley-Hamilton relation for $2 \times 2$ matrices

$$
Q_{+}^{2}-Q_{-}^{2}=Q_{+}\left\langle Q_{+}\right\rangle+\frac{1}{2}\left(\left\langle Q_{+}^{2}-Q_{-}^{2}\right\rangle-\left\langle Q_{+}\right\rangle^{2}\right)
$$

\subsection{Mass splittings to $\mathcal{O}\left(p^{2}\right)$}

At leading order, there is no mass splitting within isospin multiplets. However, at next-to-leading order the terms in the $\mathcal{O}\left(p^{2}\right)$ Lagrangians generate mass splittings. From Eqs. (12 13), we get

$$
\begin{aligned}
\Delta_{1 c}^{(2)} & \equiv\left(m_{\Sigma_{c}^{+}}-m_{\Sigma_{c}^{0}}\right)^{(2)}=2 \alpha_{1} B_{0}\left(m_{u}-m_{d}\right)+\frac{1}{6} F_{\pi}^{2} e^{2}\left(\beta_{0}-2 \beta_{4}+6 \beta_{1 h}\right), \\
\Delta_{2 c}^{(2)} & \equiv\left(m_{\Sigma_{c}^{++}}-m_{\Sigma_{c}^{0}}\right)^{(2)}=4 \alpha_{1} B_{0}\left(m_{u}-m_{d}\right)+\frac{1}{3} F_{\pi}^{2} e^{2}\left(\beta_{0}+\beta_{4}+6 \beta_{1 h}\right), \\
\Delta_{3 c}^{(2)} & \equiv\left(m_{\Xi_{c}^{\prime+}}-m_{\Xi_{c}^{\prime 0}}\right)^{(2)}=2 \alpha_{1} B_{0}\left(m_{u}-m_{d}\right)+\frac{1}{6} F_{\pi}^{2} e^{2}\left(\beta_{0}-2 \beta_{4}+6 \beta_{1 h}\right)=\Delta_{1 c}^{(2)},
\end{aligned}
$$

for the charm baryons $\Sigma_{c}$ and $\Xi_{c}^{\prime}$. Similarly, for the bottom baryons we have

$$
\begin{aligned}
\Delta_{1 b}^{(2)} & \equiv\left(m_{\Sigma_{b}^{0}}-m_{\Sigma_{b}^{-}}\right)^{(2)}=2 \alpha_{1} B_{0}\left(m_{u}-m_{d}\right)+\frac{1}{6} F_{\pi}^{2} e^{2}\left(\beta_{0}-2 \beta_{4}-3 \beta_{1 h}\right), \\
\Delta_{2 b}^{(2)} & \equiv\left(m_{\Sigma_{b}^{+}}-m_{\Sigma_{b}^{-}}\right)^{(2)}=4 \alpha_{1} B_{0}\left(m_{u}-m_{d}\right)+\frac{1}{3} F_{\pi}^{2} e^{2}\left(\beta_{0}+\beta_{4}-3 \beta_{1 h}\right), \\
\Delta_{3 b}^{(2)} & \equiv\left(m_{\Xi_{b}^{\prime 0}}-m_{\Xi_{b}^{\prime}}\right)^{(2)}=2 \alpha_{1} B_{0}\left(m_{u}-m_{d}\right)+\frac{1}{6} F_{\pi}^{2} e^{2}\left(\beta_{0}-2 \beta_{4}-3 \beta_{1 h}\right)=\Delta_{1 b}^{(2)} .
\end{aligned}
$$

Note that the $\alpha_{1}$ and $\beta_{0}$ terms always appear in the same linear combination, which means that the strong contribution, the $\alpha_{1}$ term, cannot be disentangled from the em contribution without additional information. This is completely analogous to the case of the neutron-proton mass splitting, see e.g. Ref. [4]. The $\beta_{1 h}$ term has a different sign for charm baryons and bottom baryons, hence it is expected to induce a different interference pattern of the various contributions.

From the relations given above we find

$$
\begin{aligned}
\left(m_{\Xi_{c}^{\prime+}}-m_{\Xi_{c}^{\prime 0}}\right)-\left(m_{\Sigma_{c}^{+}}-m_{\Sigma_{c}^{0}}\right) & =\mathcal{O}\left(p^{3}\right), \\
\left(m_{\Xi_{b}^{\prime 0}}-m_{\Xi_{b}^{\prime-}}\right)-\left(m_{\Sigma_{b}^{0}}-m_{\Sigma_{b}^{-}}\right) & =\mathcal{O}\left(p^{3}\right), \\
\left(m_{\Sigma_{b}^{+}}+m_{\Sigma_{b}^{-}}-2 m_{\Sigma_{b}^{0}}\right)-\left(m_{\Sigma_{c}^{++}}+m_{\Sigma_{c}^{0}}-2 m_{\Sigma_{c}^{+}}\right) & =\mathcal{O}\left(p^{3}\right) .
\end{aligned}
$$

The last relation is obtained invoking heavy quark symmetry.

\subsection{Mass splittings to $\mathcal{O}\left(p^{3}\right)$}

The first non-vanishing loop corrections to the baryon masses appear at order $\mathcal{O}\left(p^{3}\right)$. At this order formally both photon loops as well as pion-baryon loops contribute. Remarkably, QCD does

\footnotetext{
${ }^{2}$ The precise matching between two- and three-flavor versions of CHPT is discussed in Refs. 41, 42, 43.
} 


\begin{tabular}{|ll|}
\hline \hline Baryons & Loops \\
\hline$\Sigma_{c}^{++}$ & $\Sigma_{c}^{++} \pi^{0}, \Sigma_{c}^{+} \pi^{+}, \Lambda_{c}^{+} \pi^{+}$ \\
$\Sigma_{c}^{+}$ & $\Sigma_{c}^{++} \pi^{-}, \Sigma_{c}^{0} \pi^{+}, \Lambda_{c}^{+} \pi^{0}$ \\
$\Sigma_{c}^{0}$ & $\Sigma_{c}^{+} \pi^{-}, \Sigma_{c}^{0} \pi^{0}, \Lambda_{c}^{+} \pi^{-}$ \\
$\Xi_{c}^{\prime+}$ & $\Xi_{c}^{\prime+} \pi^{0}, \Xi_{c}^{\prime 0} \pi^{+}, \Xi_{c}^{+} \pi^{0}, \Xi_{c}^{0} \pi^{+}$ \\
$\Xi_{c}^{\prime 0}$ & $\Xi_{c}^{\prime+} \pi^{-}, \Xi_{c}^{\prime 0} \pi^{0}, \Xi_{c}^{+} \pi^{-}, \Xi_{c}^{0} \pi^{0}$ \\
\hline \hline
\end{tabular}

Table 1: Pion-baryon loops contributing to the charm baryon mass corrections.

not allow for a counterterm at this order and consequently the $\mathcal{O}\left(p^{3}\right)$ pieces of the these loops are finite. We start with the latter kind of loops that are to be constructed from two vertices of $\mathcal{O}(p)$. A complete list of loops is given in Table 1 for the charm baryons considered here. Since

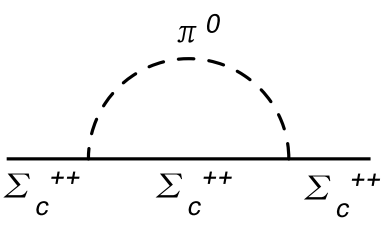

(a)

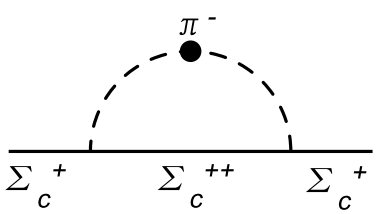

(d)

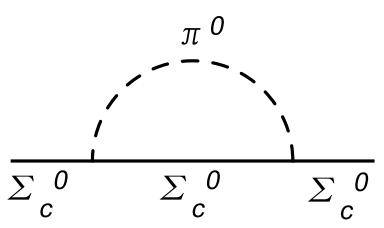

$(\mathrm{g})$

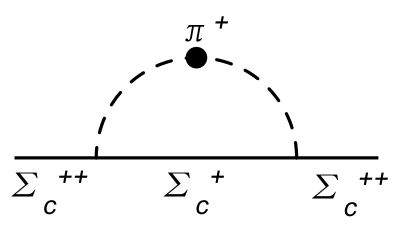

(b)

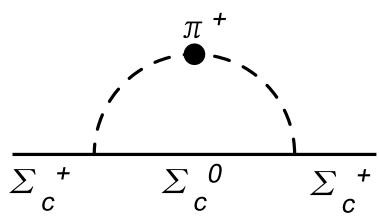

(e)

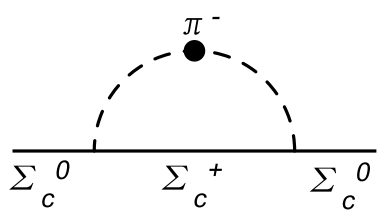

(h)

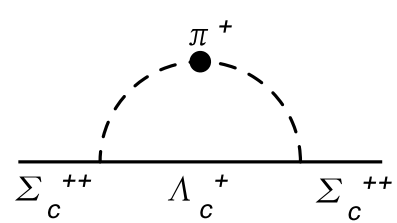

(c)

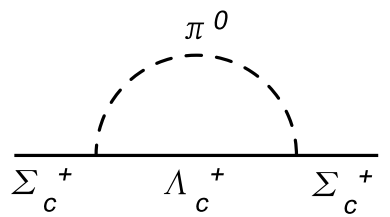

(f)

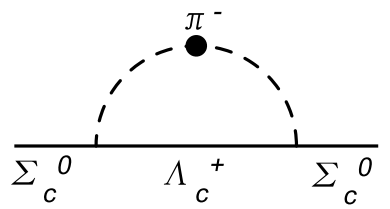

(i)

Figure 1: The pion-baryon loops contributing to the self-energies of the $\Sigma_{c}^{++}(\mathrm{a}, \mathrm{b}, \mathrm{c}), \Sigma_{c}^{+}(\mathrm{d}, \mathrm{e}$, f) and $\Sigma_{c}^{0}(\mathrm{~g}, \mathrm{~h}, \mathrm{i})$. The black dots in the charged pion propagators denote the electromagnetic insertions at $\mathcal{O}\left(p^{2}\right)$.

in the power counting the pion mass difference is of the same order as the pion mass itself, in the loops we are to use the physical pion masses (for a detailed discussion of this point, see e.g. Ref. [26]). On the other hand, to the order we are working, the masses to be used for the baryons of the same isospin multiplet are the same. Therefore, the leading $\mathrm{SU}(2)$ loop contributions to the mass corrections of the $\Xi_{c}^{\prime+}$ and $\Xi_{c}^{\prime 0}$ as well as $\Sigma_{c}^{++}$and $\Sigma_{c}^{0}$ are equal, as can be seen from Table 1. Hence there is no loop correction for the corresponding mass differences at $\mathcal{O}\left(p^{3}\right)$. The pion-baryon loops for the $\Sigma_{c}$ self-energies are shown in Fig. 1. Contrary to the case of the nucleon mass differences, here it is not straightforward to use the heavy baryon formalism to calculate the pion-baryon loops, since the pion- $\Lambda_{c}$ contribution generates a cut. It is thus more convenient for us to evaluate the integrals using the covariant method of infrared regularization as derived by 


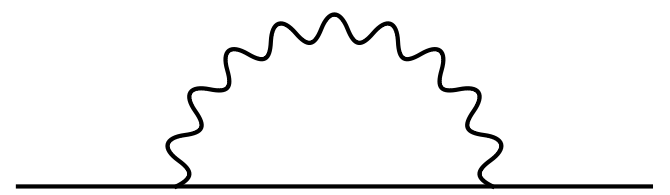

Figure 2: The photon-baryon loop.

Becher and Leutwyler [44]. Some remarks on the method and the relevant integrals are given in Appendix A.

The photon-baryon loops are shown in Fig. 2, Formally they also contribute at $\mathcal{O}\left(p^{3}\right)$. However, it can be shown that they vanish to this order. Since the baryon mass in the loop is equal to the mass of the external legs, for this loop we may use the integral representation of the heavy baryon formalism. Then the vanishing of the loop follows from the absence of a mass scale in the integral. This result also holds, when the infrared regularization is employed, as outlined in Appendix A.

Therefore the mass splittings for the charm baryons $\Sigma_{c}$ and $\Xi_{c}^{\prime}$ are to third order in the chiral expansion

$$
\begin{aligned}
& m_{\Sigma_{c}^{+}}-m_{\Sigma_{c}^{0}} \equiv \Delta_{1 c}^{(2)}+\Delta_{1 c}^{\mathrm{loop}}\left(m_{\Sigma_{c}}, m_{\Lambda_{c}}\right)+\mathcal{O}\left(p^{4}\right) \\
& m_{\Sigma_{c}^{++}}-m_{\Sigma_{c}^{0}} \equiv \Delta_{2 c}^{(2)}+\mathcal{O}\left(p^{4}\right) \\
& m_{\Xi_{c}^{\prime+}}-m_{\Xi_{c}^{\prime 0}} \equiv \Delta_{1 c}^{(2)}+\mathcal{O}\left(p^{4}\right) .
\end{aligned}
$$

The mass splittings for the bottom baryons $\Sigma_{b}$ and $\Xi_{b}^{\prime}$ are

$$
\begin{aligned}
& m_{\Sigma_{b}^{0}}-m_{\Sigma_{b}^{-}} \equiv \Delta_{1 b}^{(2)}+\Delta_{1 b}^{\text {loop }}\left(m_{\Sigma_{b}}, m_{\Lambda_{b}}\right)+\mathcal{O}\left(p^{4}\right), \\
& m_{\Sigma_{b}^{+}}-m_{\Sigma_{b}^{-}} \equiv \Delta_{2 b}^{(2)}+\mathcal{O}\left(p^{4}\right), \\
& m_{\Xi_{b}^{\prime 0}}-m_{\Xi_{b}^{\prime-}} \equiv \Delta_{1 b}^{(2)}+\mathcal{O}\left(p^{4}\right),
\end{aligned}
$$

where the results to $\mathcal{O}\left(p^{2}\right)$, the $\Delta^{(2)}$ were given in the previous subsection. As derived in Appendix $\mathrm{B}$, the loop functions to $\mathcal{O}\left(p^{3}\right)$ are given by

$$
\begin{aligned}
\Delta_{1 Q}^{\text {loop }}\left(m_{\Sigma_{Q}}, m_{\Lambda_{Q}}\right)= & -\frac{g_{1}^{2}}{32 \pi F_{\pi}^{2}}\left(M_{ \pm}^{3}-M_{0}^{3}\right) \\
& +\operatorname{Re} \Sigma^{(\mathrm{c})}\left(m_{\Sigma_{Q}} ; M_{0}, m_{\Lambda_{Q}}\right)-\operatorname{Re} \Sigma^{(\mathrm{c})}\left(m_{\Sigma_{Q}} ; M_{ \pm}, m_{\Lambda_{Q}}\right)
\end{aligned}
$$

with $M_{0}\left(M_{ \pm}\right)$the neutral (charged) pion mass. The explicit expressions for the loop functions are given in Appendix B.

\subsection{Numerical results}

When calculating the loops, we take the physical values for the masses

$$
\begin{aligned}
M_{ \pm} & =139.57 \mathrm{MeV}, \quad M_{0}=134.98 \mathrm{MeV}, \\
m_{\Sigma_{c}} & =2453.56 \mathrm{MeV}, \quad m_{\Lambda_{c}}=2286.46 \mathrm{MeV}, \\
m_{\Sigma_{b}} & =5811.5 \mathrm{MeV}, \quad m_{\Lambda_{b}}=5620.2 \mathrm{MeV} .
\end{aligned}
$$

The values of $g_{1}$ and $g_{2}$ can be estimated based on $\mathrm{SU}(6)$ [38]

$$
g_{1}=\frac{4}{3} g_{A}^{u d}, \quad g_{2}=-\sqrt{\frac{2}{3}} g_{A}^{u d},
$$


where $g_{A}^{u d}$ is the coupling constant for the single quark transition $u \rightarrow d$. A value of $g_{A}^{u d}=0.76$ gives the correct nucleon axial coupling constant $g_{A}=(5 / 3) g_{A}^{u d}=1.27$, correspondingly $g_{1}=1.02$. It also gives $g_{2}=-0.62$, the absolute value of which is close to the empirical one $\left|g_{2}\right|=0.58 \pm 0.04$ obtained from the measured decay width $\Gamma\left(\Sigma_{c}^{++} \rightarrow \Lambda_{c}^{+} \pi^{+}\right)=2.23 \pm 0.30 \mathrm{MeV}$. The only unknown parameters are the LECs in the $\mathcal{O}\left(p^{2}\right)$ Lagrangians. There are effectively three

$$
\begin{aligned}
\tilde{\gamma} & \equiv 2 \alpha_{1} B_{0}\left(m_{u}-m_{d}\right)+\frac{1}{6} e^{2} F_{\pi}^{2} \beta_{0}, \\
\tilde{\beta}_{4} & \equiv e^{2} F_{\pi}^{2} \beta_{4}, \\
\tilde{\beta}_{1 h} & \equiv e^{2} F_{\pi}^{2} \beta_{1 h} .
\end{aligned}
$$

Totally there are four known isospin mass splittings of the sextet heavy baryons 3

$$
\begin{aligned}
m_{\Sigma_{c}^{+}}-m_{\Sigma_{c}^{0}} & =-0.9 \pm 0.4 \mathrm{MeV} \\
m_{\Sigma_{c}^{++}}-m_{\Sigma_{c}^{0}} & =0.27 \pm 0.11 \mathrm{MeV}, \\
m_{\Sigma_{b}^{+}}-m_{\Sigma_{b}^{-}} & =-7.4 \pm 3.4 \mathrm{MeV}, \\
m_{\Xi_{c}^{\prime+}}-m_{\Xi_{c}^{\prime 0}} & =-2.3 \pm 4.2 \mathrm{MeV} .
\end{aligned}
$$

The first three will be taken to determine the LECs $\tilde{\gamma}, \tilde{\beta}_{4}$ and $\tilde{\beta}_{1 h}$ because they have the smallest uncertainty.

Using the physical value of the pion decay constant $F_{\pi}=92.4 \mathrm{MeV}$, we get the contribution of the loops to $m_{\Sigma_{c}^{+}}-m_{\Sigma_{c}^{0}}$

$$
\begin{aligned}
\Delta_{1 c}^{\text {loop }} & =(-0.32 \pm 0.15)+(-0.41 \pm 0.06) \mathrm{MeV} \\
& =-0.73 \pm 0.16 \mathrm{MeV}
\end{aligned}
$$

where in the first line the numbers in the first parenthesis are from the $\pi-\Sigma_{c}$ loops, and those in the second parenthesis from the $\pi-\Lambda_{c}$ loops. The uncertainty of the loops is in general controlled by the expansion parameter of CHPT, namely $\chi=M / \Lambda_{\chi}$, with the chiral symmetry breaking scale $\Lambda_{\chi} \simeq 1 \mathrm{GeV}$. Since we do not really know the value of $g_{1}$, which enters in the $\pi-\Sigma_{c}$ loops, for those we estimate the uncertainty conservatively as being of the order $4 \chi$. Since the coupling constant of the $\Sigma_{c}$ to $\Lambda_{c}$ and $\pi$ can be extracted from experiment, for the second contribution we use directly the uncertainty that results from that extraction (see above). Note that the given uncertainty is at the same time numerically of order $\chi$. Thus the uncertainty estimate is consistent with what is expected from the chiral expansion. The LECs are then determined as

$$
\begin{aligned}
\tilde{\gamma} & =-2.5 \pm 1.1 \mathrm{MeV}, \\
\tilde{\beta}_{4} & =0.6 \pm 0.9 \mathrm{MeV}, \\
\tilde{\beta}_{1 h} & =2.6 \pm 1.1 \mathrm{MeV} .
\end{aligned}
$$

The two different contributions in Eq. (30) are comparable, and they are considerably smaller than the individual strong and em contributions at $\mathcal{O}\left(p^{2}\right)$, see Eqs. (16) and (31), showing good convergence of the chiral expansion. The mass splitting within the $\Xi_{c}^{\prime}$ doublet can be predicted

$$
m_{\Xi_{c}^{\prime+}}-m_{\Xi_{c}^{\prime 0}}=m_{\Sigma_{c}^{+}}-m_{\Sigma_{c}^{0}}-\Delta_{1 c}^{\text {loop }}=-0.2 \pm 0.4(\exp ) \pm 0.4(\text { th }) \mathrm{MeV},
$$

where the first uncertainty coming from the uncertainty of $m_{\Sigma_{c}^{+}}-m_{\Sigma_{c}^{0}}$ is experimental, and the second one is theoretical. It comes from neglecting the $\mathcal{O}\left(p^{4}\right)$ contribution and was estimated 


\begin{tabular}{|c|c|c|c|c|c|c|}
\hline \hline & $\Sigma_{c}^{+}-\Sigma_{c}^{0}$ & $\Sigma_{c}^{++}-\Sigma_{c}^{0}$ & $\Xi_{c}^{\prime+}-\Xi_{c}^{\prime 0}$ & $\Sigma_{b}^{0}-\Sigma_{b}^{-}$ & $\Sigma_{b}^{+}-\Sigma_{b}^{-}$ & $\Xi_{b}^{\prime 0}-\Xi_{b}^{\prime-}$ \\
\hline Exp. [1] & $-0.9 \pm 0.4$ & $0.27 \pm 0.11$ & $-2.3 \pm 4.2$ & & $-7.4 \pm 3.4$ & \\
\hline Our work & $-0.9 \pm 0.4^{*}$ & $0.27 \pm 0.11^{*}$ & $-0.2 \pm 0.6$ & $-4.9 \pm 1.9$ & $-7.4 \pm 3.4^{*}$ & $-4.0 \pm 1.9$ \\
\hline$[8]$ & $-0.9 \pm 0.4^{*}$ & $0.27 \pm 0.11^{*}$ & & $-6.9 \pm 1.1$ & $-7.4 \pm 2.3^{*}$ & \\
{$[9]$} & -0.83 & -0.01 & -1.48 & & & \\
{$[1]$} & -0.73 & 0.28 & -3.20 & -3.95 & -6.12 & -6.16 \\
{$[10]$} & -0.2 & 1.4 & & -3.7 & -5.6 & \\
{$[12$} & -0.5 & 3.0 & -1.0 & -5.6 & -7.1 & \\
{$[13$} & -0.7 & 0.5 & -1.2 & & & \\
{$[14$} & -0.40 & 0.84 & & & & \\
{$[15$} & -0.36 & 1.20 & -0.30 & -2.51 & -3.57 & \\
{$[16$} & -0.33 & 0.37 & -0.20 & & & \\
\hline \hline
\end{tabular}

Table 2: Comparison of our results with the experimental data and the results from quark models (units are $\mathrm{MeV}$ ). The numbers marked by $*$ are used as inputs.

by taking one half of the leading loop contribution. Because the width of the $\Sigma_{b}$ has not been measured so far, $g_{2}$ for the bottom baryons cannot be determined from the data. Taking the same values as before for $g_{1}$ and $g_{2}$, as dictated by heavy quark symmetry, we get the mass difference between $\Sigma_{b}^{0}$ and $\Sigma_{b}^{-}$at $\mathcal{O}\left(p^{3}\right)$

$$
\begin{aligned}
\Delta_{1 b}^{\text {loop }} & =(-0.3 \pm 0.2)+(-0.6 \pm 0.1) \mathrm{MeV} \\
& =-0.9 \pm 0.2 \mathrm{MeV}
\end{aligned}
$$

where in the first line the numbers in the first and the second parentheses are from the $\pi-\Sigma_{b}$ loops and the $\pi-\Lambda_{b}$ loops, respectively. The uncertainties were estimated as in case of the charm baryons. According to Eq. (17), the mass of the $\Sigma_{b}^{0}$, which has not been measured yet, is predicted to be

$$
\begin{aligned}
m_{\Sigma_{b}^{0}} & =\frac{1}{2}\left(m_{\Sigma_{b}^{+}}+m_{\Sigma_{b}^{-}}-\tilde{\beta}_{4}\right)+\Delta_{1 b}^{\text {loop }} \\
& =5810.3 \pm 1.8(\exp ) \pm 0.5(\text { th }) \mathrm{MeV}
\end{aligned}
$$

We can also predict the mass difference between the $\Xi_{b}^{\prime 0}$ and $\Xi_{b}^{\prime-}$

$$
\begin{aligned}
m_{\Xi_{b}^{\prime 0}}-m_{\Xi_{b}^{\prime-}} & =\frac{1}{2}\left(m_{\Sigma_{b}^{+}}-m_{\Sigma_{b}^{-}}-\tilde{\beta}_{4}\right) \\
& =-4.0 \pm 1.8(\exp ) \pm 0.5(\text { th }) \mathrm{MeV}
\end{aligned}
$$

In Table 2, the results of our work are summarized and a comparison with those obtained in quark models is given.

\footnotetext{
${ }^{3}$ The first two mass splittings are given in PDG 1. The last two mass splittings are evaluated by taking the difference of the masses given in PDG: $m_{\Sigma_{b}^{+}}=5807.8 \pm 2.7 \mathrm{MeV}, m_{\Sigma_{b}^{-}}=5815.2 \pm 2.0 \mathrm{MeV}, m_{\Xi_{c}^{\prime+}}=2575.7 \pm 3.1 \mathrm{MeV}$, and $m_{\Xi_{c}^{\prime \prime}}=2578.0 \pm 2.9 \mathrm{MeV}$.
} 


\section{Discussions and summary}

In this work, we have calculated the mass splittings within the heavy baryon isospin multiplets $\Sigma_{c(b)}$ and $\Xi_{c(b)}^{\prime}$ to $\mathcal{O}\left(p^{3}\right)$ in the chiral expansion. Our main results are given in Eqs. (19 24) and in Table 2. To arrive at these results, we constructed both the strong and the em Lagrangians at $\mathcal{O}\left(p^{2}\right)$ which are responsible for the mass corrections. In contrast to mass splittings in light quark baryon multiplets, there is an additional operator that describes the hard virtual photons exchanged between the heavy quark and light quarks accompanied by a LEC $\beta_{1 h}$. Remarkably, this term has a different sign for the charm baryons and the bottom baryons. This is due to the fact that the sign of the electric charge of the charm quark is different from that of the bottom quark. It is the different interference between this term and the other terms that drives the mass splittings within the $\Sigma_{c}$ iso-triplet to have a different pattern compared to any other known isospin multiplet. This leads one to expect that the isospin mass splittings in the charm hadrons are always different from those in the bottom hadrons even if the heavy quark symmetry were exact. Besides the heavy baryons considered in this paper, the $D$ and $B$-meson mass splittings, $m_{D^{ \pm}}-m_{D^{0}}=4.78 \pm 0.10 \mathrm{MeV}$ and $m_{B^{0}}-m_{B^{ \pm}}=0.37 \pm 0.24 \mathrm{MeV}$ [1] are a nice example for the effect, although the ordering does not get changed here.

There is no loop contribution to the mass splitting between the two $\Xi_{c}^{\prime}$ baryons, and we predict $m_{\Xi_{c}^{\prime+}}-m_{\Xi_{c}^{\prime 0}}=-0.2 \pm 0.6 \mathrm{MeV}$. The present data for the masses of the $\Xi_{c}^{\prime}$ baryons are not accurate enough yet to test this prediction. For the $\Sigma_{b}$ states, the $\beta_{1 h}$ term interferes constructively with the other terms and hence the loop corrections are less important. The mass of the $\Sigma_{b}^{0}$ and the mass difference $m_{\Xi_{b}^{\prime 0}}-m_{\Xi_{b}^{\prime-}}$ are predicted to be $5810.3 \pm 1.9 \mathrm{MeV}$ and $-4.0 \pm 1.9 \mathrm{MeV}$, respectively, which can be tested in future experiments.

\section{Acknowledgments}

This work is partially supported by the Helmholtz Association through funds provided to the virtual institute "Spin and strong QCD" (VH-VI-231), by the EU Integrated Infrastructure Initiative Hadron Physics Project under contract number RII3-CT-2004-506078 and by DFG (SFB/TR 16, "Subnuclear Structure of Matter").

\section{A Infrared regularization}

The finite masses of the baryons in the chiral limit spoil the correspondence between the loop expansion and chiral expansion if one uses conventional dimensional regularization [45. Here we follow the infrared regularization (IR) method developed by Becher and Leutwyler [44] to overcome this problem. The IR method has been extended to two loops [46] (see also Ref. [47]), and to the cases with spin-3/2 fields [48] and spin-1 fields [49, 50] (for reviews, see [51, 52]).

In the IR method, the scalar loop integral

$$
H=\frac{1}{i} \int \frac{d^{d} k}{(2 \pi)^{d}} \frac{1}{\left(M^{2}-k^{2}\right)\left[m^{2}-(P-k)^{2}\right]} .
$$

with $M$ and $m$ being the masses of the pion and baryon, respectively, is split into two parts, one being infrared singular and the other being infrared regular, $H=I+R$. Only the singular part $I$, which is of order $\mathcal{O}(p)$, makes the expansion in loops to coincide with the chiral expansion, hence leads to a consistent power counting. The regular part $R$ can be expanded in polynomials in $M$, hence it can be absorbed into the LECs order by order. 
In any regularization such as the IR which has a consistent power counting for loops, the photon-baryon loops with each of the vertices being of the $\mathcal{O}(e)$ order should be counted as $\mathcal{O}\left(e^{2} p\right)=\mathcal{O}\left(p^{3}\right)$. In the photon-baryon loops, when taking $P^{2}=m^{2}$, which is necessary for calculating chiral corrections to the baryon mass, there is no quantity of the order of $\mathcal{O}(p)$ since the photon is massless. Therefore, the photon-baryon loops should vanish for calculating the mass shifts of baryons. Such an argument is supported by explicit calculations in the IR method.

The self-energy from any of the pion-baryon diagrams has the form

$$
-i \Sigma_{\Sigma_{Q}}^{(\mathrm{n})}(P ; M, m)=-\frac{g_{i}^{2}}{4 F_{\pi}^{2}} \int \frac{d^{d} k}{(2 \pi)^{d}} \not k \gamma_{5} \frac{i}{\left(k^{2}-M^{2}+i \varepsilon\right)} \frac{i(P-\not k+m)}{\left[(P-k)^{2}-m^{2}+i \varepsilon\right]} \not k \gamma_{5},
$$

where $(\mathrm{n})$ is a diagram label, $P$ is the external momentum, and $g_{i}(i=1,2)$ are the $\Sigma_{Q} \Sigma_{Q} \pi$ and $\Sigma_{Q} \Lambda_{Q} \pi(Q=c, b)$ coupling constants of the lowest order Lagrangian, Eq. (8). After a few manipulations one gets

$$
\Sigma_{\Sigma_{Q}}^{(\mathrm{n})}(P ; M, m)=\frac{g_{i}^{2}}{4 F_{\pi}^{2}}(\not P+m)\left[M^{2} I\left(P^{2}\right)+(m-\not P) \not P I^{(1)}\left(P^{2}\right)-\Delta_{\Sigma_{Q}}\right] .
$$

In the IR method, the single baryon loop

$$
\Delta_{\Sigma_{Q}}=i \int \frac{d^{d} k}{(2 \pi)^{d}} \frac{1}{k^{2}-m^{2}}
$$

vanishes. The expressions for the loop functions $I\left(P^{2}\right)$ and $I^{(1)}\left(P^{2}\right)$ are given in Appendix B,

Now let us focus on the $\Sigma_{c}^{++}$. Taking $P=m_{\Sigma_{c}^{++}}$, the expressions for the diagrams Fig. 1(a) and (b) are very simple because the term proportional to $(m-\not P)$ does not contribute,

$$
\Sigma^{(\mathrm{a})}\left(m_{\Sigma_{c}} ; M_{0}, m_{\Sigma_{c}}\right)=-\frac{g_{1}^{2} M_{0}^{3}}{32 \pi F_{\pi}^{2}}
$$

where the chiral limit mass $\stackrel{\circ}{m}_{\Sigma_{c}}$ in the loop has been replaced by the physical mass since the contribution of the difference is of higher order. Note that up to $\mathcal{O}\left(p^{3}\right)$, we do not need to distinguish the masses of baryons with different electric charges in loops, so that $m_{\Sigma_{c}}$, instead of $m_{\Sigma_{c}^{++}}$, is used for the arguments of the loop function. Replacing the neutral pion mass $M_{0}$ by the charged pion mass $M_{ \pm}$, the expression for diagram (b) is obtained. The contributions of the diagrams (a) and (b) do not depend on the baryon mass.

The expression for diagram (c) is much more complicated since the term proportional to $(m-P)$ has a finite contribution

$$
\Sigma^{(\mathrm{c})}\left(m_{\Sigma_{c}} ; M_{ \pm}, m_{\Lambda_{c}}\right)=\frac{g_{2}^{2}}{4 F_{\pi}^{2}}\left(m_{\Sigma_{c}}+m_{\Lambda_{c}}\right)\left[M_{ \pm}^{2} \bar{I}\left(m_{\Sigma_{c}}^{2}\right)+\left(m_{\Lambda_{c}}-m_{\Sigma_{c}}\right) m_{\Sigma_{c}} \bar{I}^{(1)}\left(m_{\Sigma_{c}}^{2}\right)\right]
$$

where the loop functions $I\left(P^{2}\right), I^{(1)}\left(P^{2}\right)$ are replaced by their finite parts $\bar{I}\left(P^{2}\right), \bar{I}^{(1)}\left(P^{2}\right)$ (subtracting the $\bar{\lambda}$ parts), and the divergences can be absorbed in the counterterms at $\mathcal{O}\left(p^{4}\right)$ which are not considered here. The expressions of the infrared singular parts of the loop integrals are given in Appendix B. Here we use the expansion of the $\bar{I}\left(P^{2}\right)$ up to $\mathcal{O}(p)$. The chiral expansion of $\bar{I}\left(P^{2}\right)$ up to $\mathcal{O}(p)$, accounting for the cut due to the opening of the $\Lambda_{c} \pi$ channel, is

$$
\bar{I}\left(P^{2}\right)=-\frac{\alpha}{16 \pi^{2}}\left\{\Omega(2 \ln \alpha-1)+\sqrt{\Omega^{2}-1}\left[\ln \left(\frac{\Omega+\sqrt{\Omega^{2}-1}}{\Omega-\sqrt{\Omega^{2}-1}}\right)-2 i \pi\right]\right\}+O\left(\alpha^{2}\right),
$$


where

$$
\alpha=\frac{M_{ \pm}}{m_{\Lambda_{c}}}, \quad \Omega=\frac{P^{2}-m_{\Lambda_{c}}^{2}-M_{ \pm}^{2}}{2 M_{ \pm} m_{\Lambda_{c}}} .
$$

For $P^{2}-m_{\Lambda_{c}}^{2} \sim \mathcal{O}(p)$, which is the case for taking $P^{2}=m_{\Sigma_{c}}^{2}$ since $m_{\Sigma_{c}}-m_{\Lambda_{c}} \simeq 170 \mathrm{MeV}$, $I^{(1)}\left(P^{2}\right)$ starts from $\mathcal{O}\left(p^{2}\right)$, see Eq. (B.6). The physical mass of the $\Sigma_{c}$ is above the $\Lambda_{c} \pi$ threshold, correspondingly $\Omega>1$.

Summing up the diagrams (a), (b) and (c), one gets the corrections to the mass of the $\Sigma_{c}^{++}$at $\mathcal{O}\left(p^{3}\right)$

$$
\Delta m_{\Sigma_{c}^{++}}^{\text {loop }}=-\frac{g_{1}^{2}\left(M_{0}^{3}+M_{ \pm}^{3}\right)}{32 \pi F_{\pi}^{2}}+\operatorname{Re} \Sigma^{(\mathrm{c})}\left(m_{\Sigma_{c}} ; M_{ \pm}, m_{\Lambda_{c}}\right)
$$

where Re represents taking the real part. Similarly for the $\Sigma_{c}^{+}$and $\Sigma_{c}^{0}$, we have

$$
\begin{aligned}
& \Delta m_{\Sigma_{c}^{+}}^{\text {loop }}=-\frac{2 g_{1}^{2} M_{ \pm}^{3}}{32 \pi F_{\pi}^{2}}+\operatorname{Re} \Sigma^{(\mathrm{c})}\left(m_{\Sigma_{c}} ; M_{0}, m_{\Lambda_{c}}\right), \\
& \Delta m_{\Sigma_{c}^{0}}^{\text {loop }}=\Delta m_{\Sigma_{c}^{++}}^{\text {loop }} .
\end{aligned}
$$

\section{B Loop integrals}

Defining

$$
\begin{aligned}
& \alpha=\frac{M}{m}, \quad s=P^{2}, \quad \Omega=\frac{s-m^{2}-M^{2}}{2 M m}, \\
& \bar{\lambda}=\frac{m^{d-4}}{(4 \pi)^{2}}\left[\frac{1}{d-4}-\frac{1}{2}\left(\ln (4 \pi)+\Gamma^{\prime}(1)+1\right)\right],
\end{aligned}
$$

the infrared singular part of the loop integral

$$
H(s)=\frac{1}{i} \int \frac{d^{d} k}{(2 \pi)^{d}} \frac{1}{\left(M^{2}-k^{2}\right)\left[m^{2}-(P-k)^{2}\right]},
$$

is (the expression for $-1<\Omega<1$ is given in Ref. [44])

$$
\begin{aligned}
& I(s)=\bar{I}(s)-\frac{s-m^{2}+M^{2}}{s} \bar{\lambda} \\
& \bar{I}(s)=-\frac{1}{16 \pi^{2}} \frac{\alpha}{1+2 \alpha \Omega+\alpha^{2}}[(\Omega+\alpha)(2 \ln \alpha-1)+F(s)],
\end{aligned}
$$

where

$$
F(s)= \begin{cases}\sqrt{\Omega^{2}-1}\left[\ln \left(-\sqrt{\Omega^{2}-1}-\Omega-\alpha\right)-\ln \left(\sqrt{\Omega^{2}-1}-\Omega-\alpha\right)\right], & \Omega<-1, \\ 2 \sqrt{1-\Omega^{2}} \arccos \left(-\frac{\Omega+\alpha}{\sqrt{1+2 \alpha \Omega+\alpha^{2}}}\right), & -1<\Omega<1, \\ \sqrt{\Omega^{2}-1}\left[\ln \left(\sqrt{\Omega^{2}-1}+\Omega+\alpha\right)-\ln \left(\Omega+\alpha-\sqrt{\Omega^{2}-1}\right)-2 i \pi\right], & \Omega>1 .\end{cases}
$$

In a more compact way, one can rewrite this by keeping the $i \varepsilon, \varepsilon \rightarrow 0^{+}$, explicitly, which is necessary to choose the correct Riemann sheet,

$$
F(s)=\sqrt{\Omega^{2}-1}\left[\ln \left(-\sqrt{\Omega^{2}-1}-\Omega-\alpha-i \varepsilon\right)-\ln \left(\sqrt{\Omega^{2}-1}-\Omega-\alpha+i \varepsilon\right)\right] .
$$


$I^{(1)}(s)$ is defined as

$$
P^{\mu} I^{(1)}(s)=\frac{1}{i} \int \frac{d^{d} k}{(2 \pi)^{d}} \frac{k^{\mu}}{\left(M^{2}-k^{2}\right)\left[m^{2}-(P-k)^{2}\right]} .
$$

One gets

$$
I^{(1)}(s)=\frac{1}{2 s}\left[\left(s-m^{2}+M^{2}\right) I(s)+\Delta_{\pi}-\Delta_{\Sigma_{Q}}\right],
$$

where in the IR method [44,

$$
\begin{aligned}
\Delta_{\pi} & =i \int \frac{d^{d} k}{(2 \pi)^{d}} \frac{1}{k^{2}-M^{2}}=2 M^{2}\left(\bar{\lambda}+\frac{1}{16 \pi^{2}} \ln \alpha\right), \\
\Delta_{\Sigma_{Q}} & =i \int \frac{d^{d} k}{(2 \pi)^{d}} \frac{1}{k^{2}-m^{2}}=0 .
\end{aligned}
$$

\section{References}

[1] C. Amsler et al. [Particle Data Group], Phys. Lett. B 667 (2008) 1.

[2] T. Aaltonen et al. [CDF Collaboration], Phys. Rev. Lett. 99 (2007) 202001 arXiv:0706.3868 [hep-ex]].

[3] J. Gasser and H. Leutwyler, Annals Phys. 158 (1984) 142.

[4] U.-G. Meißner and S. Steininger, Phys. Lett. B 419 (1998) 403 arXiv:hep-ph/9709453.

[5] G. Müller and U.-G. Meißner, Nucl. Phys. B 556 (1999) 265 arXiv:hep-ph/9903375.

[6] S. Weinberg, Trans. New York Acad. Sci. 38 (1977) 185.

[7] J. Gasser, Annals Phys. 136 (1981) 62.

[8] C. W. Hwang and C. H. Chung, arXiv:0804.4044 [hep-ph].

[9] K. P. Tiwari, C. P. Singh and M. P. Khanna, Phys. Rev. D 31 (1985) 642.

[10] S. Capstick, Phys. Rev. D 36 (1987) 2800.

[11] L. H. Chan, Phys. Rev. D 31 (1985) 204.

[12] W. Y. P. Hwang and D. B. Lichtenberg, Phys. Rev. D 35 (1987) 3526.

[13] R. C. Verma and S. Srivastava, Phys. Rev. D 38 (1988) 1623.

[14] R. E. Cutkosky and P. Geiger, Phys. Rev. D 48 (1993) 1315 arXiv:hep-ph/9304202.

[15] K. Varga, M. Genovese, J. M. Richard and B. Silvestre-Brac, Phys. Rev. D 59 (1999) 014012 [arXiv:hep-ph/9803340].

[16] B. Silvestre-Brac, F. Brau and C. Semay, J. Phys. G 29 (2003) 2685 arXiv:hep-ph/0302252.

[17] R. Urech, Nucl. Phys. B 433 (1995) 234 arXiv:hep-ph/9405341. 
[18] U.-G. Meißner, G. Müller and S. Steininger, Phys. Lett. B 406 (1997) 154 [Erratum-ibid. B 407 (1997) 454] arXiv:hep-ph/9704377.

[19] M. Knecht and R. Urech, Nucl. Phys. B 519 (1998) 329 arXiv:hep-ph/9709348].

[20] N. Fettes and U.-G. Meißner, Nucl. Phys. A 693 (2001) 693 arXiv:hep-ph/0101030].

[21] B. Kubis and U.-G. Meißner, Nucl. Phys. A 671 (2000) 332 [Erratum-ibid. A 692 (2001) 647] arXiv:hep-ph/9908261].

[22] G. Amoros, J. Bijnens and P. Talavera, Nucl. Phys. B 602 (2001) 87 arXiv:hep-ph/0101127.

[23] B. Kubis and U.-G. Meißner, Nucl. Phys. A 699 (2002) 709 arXiv:hep-ph/0107199].

[24] A. Nehme and P. Talavera, Phys. Rev. D 65 (2002) 054023 arXiv:hep-ph/0107299.

[25] M. Knecht and A. Nehme, Phys. Lett. B 532 (2002) 55 arXiv:hep-ph/0201033.

[26] J. Gasser, M. A. Ivanov, E. Lipartia, M. Mojzis and A. Rusetsky, Eur. Phys. J. C 26 (2002) 13 arXiv:hep-ph/0206068.

[27] J. Schweizer, JHEP 0302 (2003) 007 [arXiv:hep-ph/0212188].

[28] J. Gasser, A. Rusetsky and I. Scimemi, Eur. Phys. J. C 32 (2003) 97 arXiv:hep-ph/0305260.

[29] J. Bijnens and K. Ghorbani, arXiv:0711.0148 [hep-ph].

[30] F. K. Guo, C. Hanhart, S. Krewald and U.-G. Meißner, Phys. Lett. B 666 (2008) 251 arXiv:0806.3374 [hep-ph]].

[31] J. Bijnens, H. Sonoda and M. B. Wise, Nucl. Phys. B 261 (1985) 185.

[32] B. Borasoy and U.-G. Meißner, Annals Phys. 254 (1997) 192 arXiv:hep-ph/9607432.

[33] J. F. Donoghue, B. R. Holstein and B. Borasoy, Phys. Rev. D 59 (1999) 036002 arXiv:hep-ph/9804281.

[34] A. Roessl, Nucl. Phys. B 555 (1999) 507 arXiv:hep-ph/9904230.

[35] M. Frink, B. Kubis and U.-G. Meißner, Eur. Phys. J. C 25 (2002) 259 arXiv:hep-ph/0203193.

[36] S. R. Beane, P. F. Bedaque, A. Parreno and M. J. Savage, Nucl. Phys. A 747 (2005) 55 arXiv:nucl-th/0311027.

[37] B. C. Tiburzi and A. Walker-Loud, arXiv:0808.0482 [nucl-th].

[38] T. M. Yan, H. Y. Cheng, C. Y. Cheung, G. L. Lin, Y. C. Lin and H. L. Yu, Phys. Rev. D 46 (1992) 1148 [Erratum-ibid. D 55 (1997) 5851].

[39] H. Y. Cheng, C. Y. Cheung, G. L. Lin, Y. C. Lin, T. M. Yan and H. L. Yu, Phys. Rev. D 49 (1994) 5857 [Erratum-ibid. D 55 (1997) 5851] [arXiv:hep-ph/9312304].

[40] P. L. Cho, Phys. Lett. B 285 (1992) 145 arXiv:hep-ph/9203225.

[41] J. Gasser and H. Leutwyler, Nucl. Phys. B 250 (1985) 465.

[42] M. Frink and U.-G. Meißner, JHEP 0407 (2004) 028 arXiv:hep-lat/0404018. 
[43] J. Gasser, C. Haefeli, M. A. Ivanov and M. Schmid, Phys. Lett. B 652 (2007) 21 arXiv:0706.0955 [hep-ph]].

[44] T. Becher and H. Leutwyler, Eur. Phys. J. C 9 (1999) 643 arXiv:hep-ph/9901384.

[45] J. Gasser, M. E. Sainio and A. Svarc, Nucl. Phys. B 307 (1988) 779.

[46] M. R. Schindler, D. Djukanovic, J. Gegelia and S. Scherer, Nucl. Phys. A 803 (2008) 68 arXiv:0707.4296 [hep-ph]].

[47] D. Lehmann and G. Prezeau, Phys. Rev. D 65 (2002) 016001 arXiv:hep-ph/0102161.

[48] V. Bernard, T. R. Hemmert and U.-G. Meißner, Phys. Lett. B 565 (2003) 137 arXiv:hep-ph/0303198.

[49] P. C. Bruns and U.-G. Meißner, Eur. Phys. J. C 40 (2005) 97 arXiv:hep-ph/0411223.

[50] P. C. Bruns and U.-G. Meißner, arXiv:0808.3174 [hep-ph].

[51] S. Scherer, Adv. Nucl. Phys. 27 (2003) 277 [arXiv:hep-ph/0210398].

[52] V. Bernard, Prog. Part. Nucl. Phys. 60 (2008) 82 [arXiv:0706.0312 [hep-ph]]. 